\title{
Assessing Ghanaian Teachers' Perceptions and Beliefs Regarding Substance Use Among School-Aged Children in Ghana
}

\author{
Theresa Marie Hunter,," Cecilia Obeng, ${ }^{1}$ and Joseph Ogah ${ }^{2}$ \\ ${ }^{1}$ School of Public Health, Indiana University, Indiana, USA \\ ${ }^{2}$ Cape Coast University, Cape Coast, Ghana \\ "Corresponding author: Theresa Marie Hunter, School of Public Health, Indiana University, Indiana, USA. Tel: +1-2195120120, E-mail: tmhunter@indiana.edu
}

Received 2016 February 28; Revised 2016 May 01; Accepted 2016 June 02.

\begin{abstract}
Background: Substance use among school-aged children is an international public health concern. Rates of tobacco, alcohol, and illicit drug use among school children in Ghana have been increasing.

Objectives: The objective of this study was to assess Ghanaian teachers' beliefs and perceptions regarding substance use among school children in Ghana.

Methods: A cross-sectional design was used. A sample of teachers from the Central and Ashanti Regions of Ghana completed a survey questionnaire assessing their perceptions of substance use among Ghanaian school children. Data were analyzed using SPSS statistical software version 23.0. T-tests and chi-square tests were used to identify associations between participants' demographic factors and beliefs regarding drug use among school-aged children.

Results: The sample consisted of 289 teachers with over half (57.7\%) of them being male, and ages ranging from 20-66 years old. The majority of the teachers (50.9\%) indicated that they believed alcohol was the most common substance used by school-aged children in Ghana. Over $90 \%$ of them $\mathrm{m}$ indicated that they believe school children in Ghana will first use alcohol before they are 13 years old. Only 54.3\% of teachers indicated that they are aware of drug policies in the schools where they teach.

Conclusions: The results from this study should encourage the development and implementation of school policies on substance use and health education programs that discuss the risks and circumstances of substance use.
\end{abstract}

Keywords: Substance Use, School, Ghana, Teachers

\section{Background}

Substance use among school-aged children is a serious international public health concern. Similar to the increase of substance use in the United States, many African countries are experiencing a rise in tobacco, alcohol, and illicit drug use among adolescents. Results from the global School-based health survey questionnaire, indicated a prevalence of tobacco use (12.6\%), alcohol use (6.6\%), and of illicit drug use (10.5\%) in school-going adolescents in six African countries Kenya, Namibia, Swaziland, Uganda, Zambia, and Zimbabwe (1).

Ghana, like these other African nations, has experienced an increase in the use of substances such as tobacco, alcohol, and marijuana among adolescents in recent years. According to the 2012 Ghana junior high global-based student health survey (GSHS), 15.4\% of students stated that they drank at least one drink containing alcohol on one or more occasion during the last 30 days (2). Additionally, 9.4\% of students stated that they drank so much alcohol that they were intoxicated one or more times during their lives (2). While only 8.3\% of students stated that they had smoked cigarettes within the last 30 days, $94.3 \%$ of stu- dents had tried cigarettes before the age of 14 (2).

Schools serve as a physical, social, and psychological home away from home for adolescents. In addition, school teachers and administrators often serve as role models for their students. Given this, schools naturally assume a role in substance use education, prevention, and early identification (3). Schools are well situated to identify students with signs and symptoms of illicit drug abuse $(4,5)$. The degree of illicit substance abuse among students has translated into an ongoing quest for ways to address this problem, including community- and school-based prevention programs (3).

With these increasing rates of alcohol and drug use among school-aged children in Ghana, it is important to understand the perceptions and beliefs of school teachers regarding this epidemic. Teachers are in a position to educate students on the harms of substance use, but are also able to identify and help students who are in need of resources and interventions. Furthermore, given that Ghanaian teachers spend many hours interacting with school-aged children on a daily basis, it is important to understand their point of view on the substance use problem. 


\section{Objectives}

The objective of this study, therefore, was to assess Ghanaian teachers' beliefs and perceptions regarding substance use among school children in Ghana.

\section{Methods}

\subsection{Study Sample}

A convenience sample $(n=289)$ of teachers were recruited from public schools in the Central and Ashanti Regions of Ghana. Based on Cohen's primer to determine sample size, the estimated total sample size of $n=289$ was determined assuming power of $90 \%$ with $\alpha=0.05$ to determine a small effect for a multiple regression analysis. Teachers were recruited from both primary and secondary schools in Ghana. They were made fully aware that participating in the survey was completely voluntary and confidential. The study was approved by the Indiana university institutional review board.

\subsection{Data Collection}

A self-administered questionnaire was used in this study. The survey instrument included demographic factors such as age, sex, educational level, and relationship status. Items to assess beliefs regarding drug use among school-aged children were also included. The instrument format incorporated dichotomous, categorical, ordinal, and scale items as well as open-ended questions. The study questionnaire was pilot tested and tested for content validity and test-retest reliability $(\alpha=0.71)$.

\subsection{Data Analysis}

Data were analyzed using SPSS statistical software version 23.0. This software was used for descriptive statistics where data is shown as percentages, means, and standard deviations to show demographic information. T-tests and chi-square tests were used to identify associations between participants' demographic factors and beliefs regarding drug use among school-aged children. Frequency distributions were used to test normality for each of these variables.

\section{Results}

\subsection{Demographic Characteristics}

The sample consisted of 289 teachers with over half (57.7\%) of them being male. The ages of the teachers ranged from 20-66 years. Age was divided into four groups: 20 - 29 (21.7\%), 30 - 39 (53.6\%), 40 - 49 (18.8\%) and $\geq 50$ (5.8\%) years. The majority of teachers were single (63.7\%) and $83.3 \%$ had earned at least a bachelor's degree. Class sizes for these teachers ranged from 12 students in a class to a total of 660 students in all the classes. A full description of the demographic information is shown in Table 1.

Table 1. Descriptive Characteristics of Participants

\begin{tabular}{|c|c|c|}
\hline Characteristic & No. $(n=289)$ & Percent (\%) \\
\hline \multicolumn{3}{|l|}{ Sex } \\
\hline Male & 167 & 57.7 \\
\hline Female & 122 & 42.3 \\
\hline \multicolumn{3}{|l|}{ Age, $y$} \\
\hline $20-29$ & 60 & 21.7 \\
\hline $30-39$ & 148 & 53.6 \\
\hline $40-49$ & 52 & 18.8 \\
\hline$\geq 50$ & 16 & 5.8 \\
\hline \multicolumn{3}{|l|}{ Education Level } \\
\hline Senior High/ Technical School & 2 & 0.7 \\
\hline Certificate & 2 & 0.7 \\
\hline Diploma & 41 & 14.2 \\
\hline Bachelor Degree & 190 & 65.7 \\
\hline Master Degree & 51 & 17.6 \\
\hline \multicolumn{3}{|l|}{ Relationship Status } \\
\hline Single: Not Married & 184 & 63.7 \\
\hline Single: In a relationship & 47 & 16.3 \\
\hline Married & 24 & 8.3 \\
\hline Separated/Divorced & 29 & 10.0 \\
\hline Widowed & 2 & 0.7 \\
\hline
\end{tabular}

\subsection{Substance Use}

The majority of the respondents (50.9\%) indicated that they believed alcohol was the most common substance used by school-aged children in Ghana. This was followed by inhalants (26.3\%), cigarettes (10.0\%), and marijuana (10.0\%). Of the 289 teachers in this study, 206 indicated that they believe at least $50 \%$ of school-aged children in Ghana use alcohol or other drugs.

Pearson's chi-squared test was conducted to determine whether the teachers' beliefs regarding substance use among school children in Ghana differed by varying education levels, sex, and age. The respondents' belief regarding the proportion of school-aged children in Ghana that use substances was significantly different by $\operatorname{sex}(\mathrm{P}=0.049)$ and age $(\mathrm{P}=0.001)$. While education level was not significantly associated with the beliefs regards the proportion of school-aged children who used substances, education level 
was significantly associated to whether or not the teachers believed that children used drugs at school $(\mathrm{P} \leq 0.001)$.

\subsection{Age at First Use of Substance}

Teachers' beliefs regarding the age at which school children in Ghana first use substances varied by each specific substance. Over 90\% of teachers indicated that they believe school children in Ghana will first use alcohol before they are 13 years old (Figure 1 ). In regards to cigarette use, $69.5 \%$ of teachers believed that school children in Ghana will first use cigarettes between the ages of 11 - 14 years, while $24.6 \%$ believed that school children in Ghana will first use cigarettes before they are 11 years old. The majority (62.3\%) of teachers indicated that they believe school children in Ghana will first use marijuana between the ages of $13-14$, while over half (50.5\%) believed that school children will not use inhalants until they are at least 15 years old.

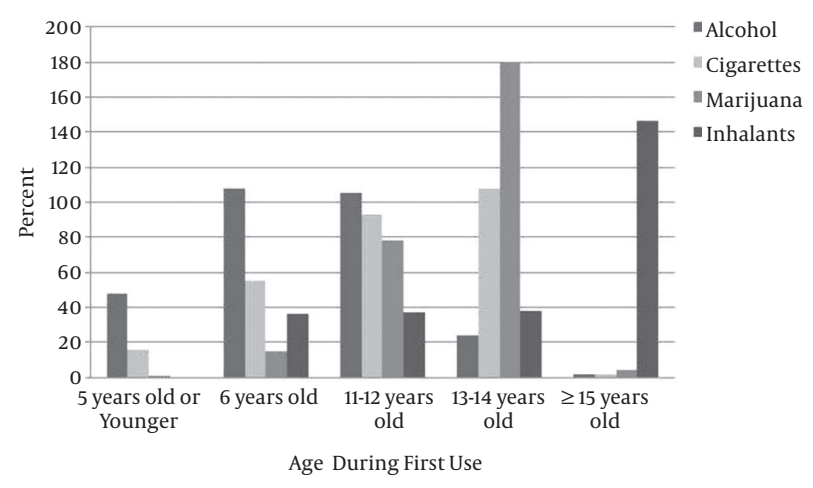

Figure 1. Age at Which Teacher's Believe Students First Use Substances

Pearson's chi-squared tests were performed to identify potential differences in beliefs regarding the age of a child's first use and the sex, age, and education level of the teachers. In regards to teachers' beliefs on the age at which school children in Ghana first use alcohol, there were significant differences between these beliefs and the age $(\mathrm{P}=$ $0.043)$, sex $(P=0.012)$, and education level $(P \leq 0.001)$ of the teachers. Teachers who were male, under the age of 30 and did not have a college degree were more likely to state that children first use alcohol before the age of 10 year old, while teachers who are female, over the age of 30 who at least had a bachelor's degree indicated that children will first use alcohol between the ages of $11-14$. The age of the teachers was also significantly associated with their beliefs regarding the age of cigarette use $(\mathrm{P}=0.040)$, with younger teachers believing that children use cigarettes before the age of 10 .
In regards to respondents' beliefs on the age at which school children first use marijuana, there were significant differences between beliefs and the $\operatorname{sex}(\mathrm{P}=0.017)$ and education level $(\mathrm{P} \leq 0.001)$ of the teacher. Male teachers and teachers with a graduate-level college degree were more likely to believe that children first use marijuana when they are 13-14 years old, whereas female teachers and teachers without a college degree were more likely to believe that children in Ghana first use marijuana at a younger age.

\subsection{Environmental Factors Influencing Substance Use}

To better understand the environmental factors that influence substance use among school children in Ghana, the teachers answered various questions to assess the impact of peers, media, and school policies. A majority of the teachers $(67.1 \%)$ indicated that they believe that peers influence school children in Ghana to use drugs, followed by media (25.3\%), families (5.5\%), and culture (1.7\%). In regards to drug use at school, $87.5 \%$ of teachers indicated that children are likely to use drugs while at school. Only $54.3 \%$ indicated that they were aware of drug policies in the schools where they teach.

\section{Discussion}

In today's society, students spend as much time at school as they do at other places such as home and work. Since so much of a student's time is spent in the classroom, it is important to understand how informed teachers are regarding the challenges and health concerns that their students face and/or endure. The purpose of this study was to assess Ghanaian teachers' beliefs and perceptions regarding substance use among school children. The findings from this study provide insights into the knowledge and awareness of substance abuse issues among Ghanaian school teachers.

While the beliefs and knowledge regarding substance use varied depending on the teachers' age, sex, and education level, the overall knowledge regarding substance use prevalence in Ghana among the teachers was similar to previous findings. Over $90 \%$ of teachers in this study indicated that they believe school children in Ghana will first use alcohol before they are 13 years old. This finding is similar to the results of the 2012 Ghana junior high globalbased student health survey, which found that among students who ever had a drink of alcohol, 86.1\% had their first drink before the age of 14 (2).

Adolescence is a vital period for developing pattern of substance use and many times, these patterns continue into adulthood (6). While the use of marijuana and illicit drugs are illegal in Ghana, alcohol is legal (for adults, 18 
years and over) and easily assessable and available to minors (7). The initial stages of substance use typically include experimentation with alcohol and tobacco during the early and middle years of adolescence (8). It is important to implement programs to decrease the rates of early initiation of substances. Research has shown that experimentation with alcohol and drugs during elementary or primary school significantly increase the risk for more serious drug problems in later years $(9,10)$.

The teachers in this study stated that they believed that students would first use cigarettes and alcohol before the age of 13 . In regards to marijuana and inhalants use, the majority of teachers indicated that students would not try these substances until they were in their later teen years. These findings are supported by previous research by Obot (11) and Odejide (12), which found that reports from school surveys in countries across Africa show that adolescents' use and abuse of substances will start with alcohol and cigarettes. Those who initiate alcohol use before age 15 years are 4 times as likely to develop alcohol dependence compared to those who wait unto they are 21 years or older (13). These findings emphasize the need for health education programs and preventative efforts need to be implemented in elementary schools to help reduce early substance use $(14,15)$.

While the findings support the notion that the majority of Ghanaian teachers are aware of the substance use issues that school-aged children in Ghana face, they also support the conclusion that more efforts need to be done to address this problem. Among the 289 teachers who participated in this survey, over $50 \%$ were not aware of substance use policies in the schools where they work. It is important that teachers, school officials, and administrators are not only aware of the substance use issues among school children, but that they are also helping to address these issues inside and outside of the classroom.

\subsection{Conclusion}

When comparing the findings of this study to those of the previous literature, it is important to note that many of the teachers' beliefs and perceptions regarding substance use among school-aged children in Ghana are aligned with findings from the Ghana Junior high global-based student health survey (2). While many teachers are aware of the substance use issues in Ghana, many schools have not taken a proactive approach to addressing these issues. The results from this study should encourage the development and implementation of school policies on substance use and health education programs that discuss the risks and circumstances of substance use.

\section{Footnote}

Authors' Contribution: Study concept and design, Cecilia Obeng, Joseph Ogah, Theresa Marie Hunter; acquisition of data, Cecilia Obeng, Joseph Ogah; analysis and interpretation of data, Theresa Marie Hunter, Cecilia Obeng, Joseph Ogah; drafting of the manuscript, Theresa Marie Hunter; critical revision of the manuscript for important intellectual content, Cecilia Obeng, Joseph Ogah; statistical analysis, Theresa Marie Hunter; administrative, technical, and material support, Theresa Marie Hunter, Cecilia Obeng, Joseph Ogah; Study supervision, Cecilia Obeng, Joseph Ogah,

\section{References}

1. Peltzer K. Prevalence and correlates of substance use among school children in six African countries. Int J Psychol. 2009;44(5):378-86.

2. Ghana Junior . Global school-based student health survey 2012. Available from: http://www.who.int/chp/gshs/2012_Ghana_junior_ high_fact_sheet.pdf?ua=1.

3. American academy of pediatrics . The role of schools in combating substance abuse. Pediatrics. 1995;95(5):784-5.

4. Flay BR. Approaches to substance use prevention utilizing school curriculum plus social environment change. Addictive behav. 2000;25(6):861-85.

5. Cuijpers P. Effective ingredients of school-based drug prevention programs: A systematic review. Addictive Behav. 2002;27(6):1009-23.

6. Botvin GJ, Griffin KW. School-based programmes to prevent alcohol, tobacco and other drug use. Int Rev psychiat. 2007;19(6):607-15.

7. Adu-Mireku S. The prevalence of alcohol, cigarette, and marijuana use among Ghanaian senior secondary students in an urban setting. Ethnicity Substance Abuse. 2003;2(1):53-65.

8. Kandel DB. Stages and pathways of drug involvement: Examining the gateway hypothesis. Cambridge University Press; 2002.

9. Anthony JC, Petronis KR. Early-onset drug use and risk of later drug problems. Drug Alcohol Depend. 1995;40(1):9-15.

10. Wilson N, Battistich V, Syme SL, Boyce W. Does elementary school alcohol, tobacco, and marijuana use increase middle school risk?. Adolescent Health. 2002;30(6):442-7.

11. Obot IS. Responding to substance use problems in Nigeria: The role of civil society organizations. Substance Use Misuse. 2004;39(8):1287-99.

12. Odejide AO. Status of drug use/abuse in Africa: A review. Int J Mental Health Addiction. 2006;4(2):87-102.

13. Grant BF, Dawson DA. Age at onset of alcohol use and its association with dsm-iv alcohol abuse and dependence: Results from the national longitudinal alcohol epidemiologic survey. Substance Abuse. 1997;9:103-10.

14. Lloyd C, Joyce R, Hurry J, Ashton M. The effectiveness of primary school drug education. Drugs: education, prevention and policy. 2000;7(2):109-26.

15. Botvin GJ, Griffin KW, Paul E, Macaulay AP. Preventing tobacco and alcohol use among elementary school students through life skills training. Child Adolescent Substance Abuse. 2003;12(4):1-17. 